
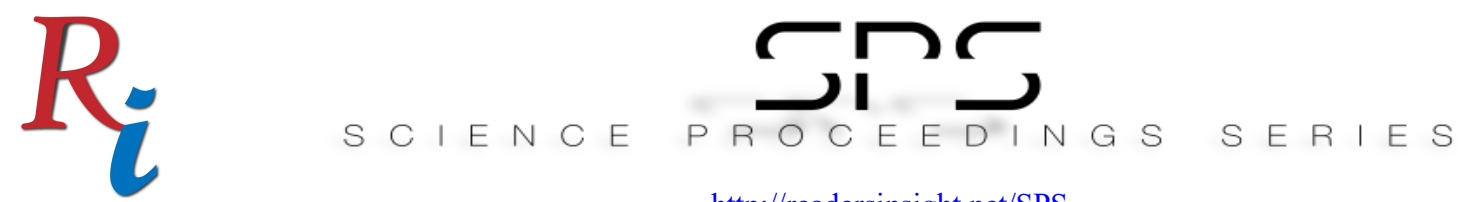

http://readersinsight.net/SPS

\title{
Structure and Dynamics of Spin Label Side Chains In KvAP Voltage-Sensing Domain: An All-ATOM MD Simulation Study
}

Ngoc Lan Le Nguyen

Department of Chemistry

Chulalongkorn University

Thailand

lenguyenngoclan95@gmail.com

\section{Pornthep Sompornpisut*}

Department of Chemistry

Chulalongkorn University

Thailand

Pornthep.S@chula.ac.th

*Corrosponding author's Email: Pornthep.S@chula.ac.th 


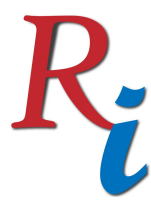

A b s t r a c t

Spin labeling technique in combination with continuous-wave electron paramagnetic resonance spectroscopy has become a useful approach to investigate the structure and dynamics of biomolecules, especially membrane protein. In this method, spin label side chains due to their motion highly depending on the local environment were employed as external molecules to explore the structure and dynamics of the membrane protein. The close relationships between the spin label side chain dynamical feature and protein structure have been investigated for T4 lysozyme. Besides, molecular dynamics (MD) simulations were widely applied as an effective approach to study the microscopic behavior of the proteins, completing experimental approaches. Here, we presented an atomistic MD simulation study of the nitroxide spin label (MTSSL) side chains in a potassium ion channel voltage-sensing domain (VSD) from Aeropyrum Pernix (KvAP-VSD).

Keywords: Membrane Protein, Spin Labeling, MD simulations, MTSSL, KvAP

\section{Rese a r ch H i g h I igh t s}

- This work is one of the pioneer computational investigations applying SDSL for all residues in a membrane protein and performing MD simulations.

- The dynamics of spin label side chains were well consistent with the experimental EPR data.

- Spin label side chain can reflect the conformational changes and flexibilities of membrane protein. 
$R_{i}$

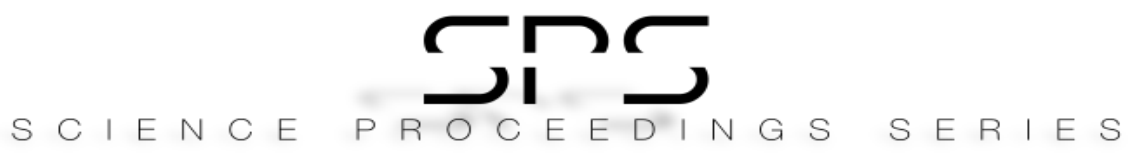

http://readersinsight.net/SPS

\section{Graphical A bst ract}
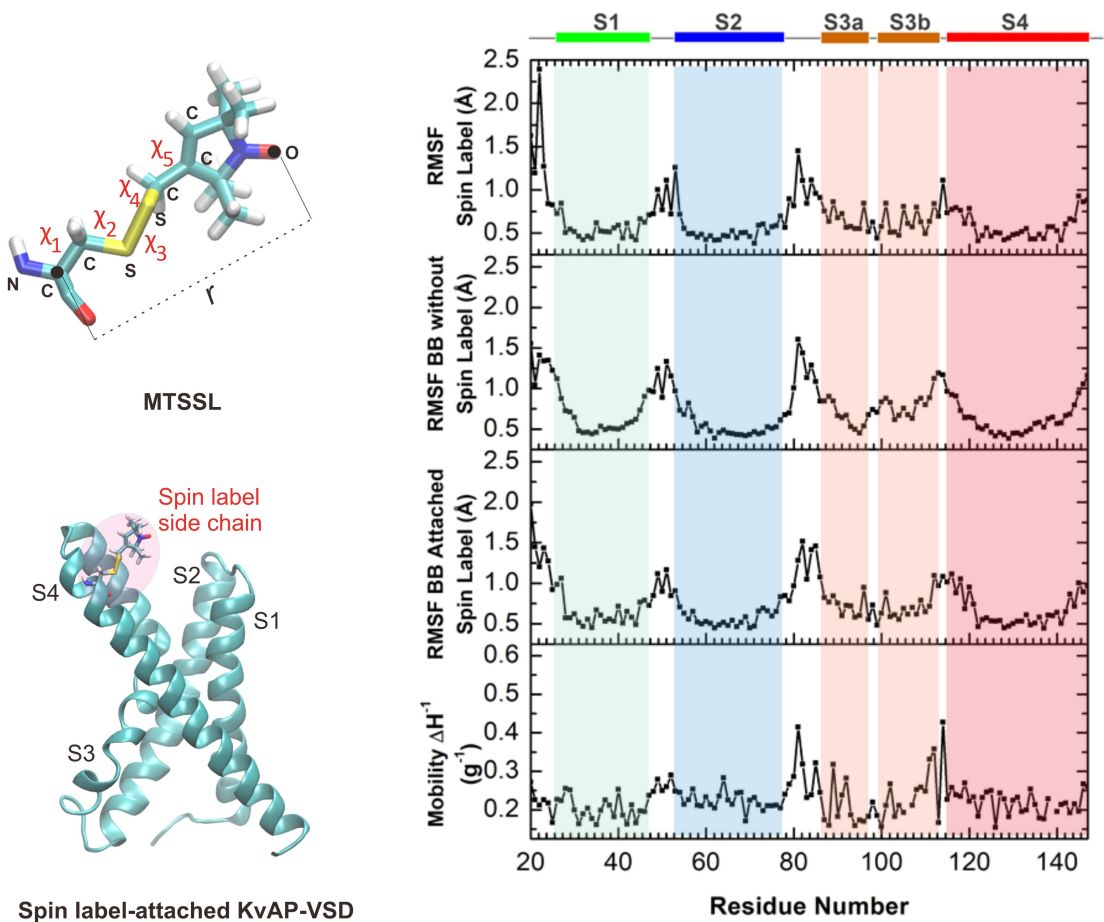

Spin label-attached KvAP-VSD

\section{Research Objectives}

In this research, we aim to model structures of cysteine-mutated membrane protein with nitroxide spin label attaching at various positions and then investigate typical structural and dynamical features of spin label in membrane protein.

\section{Methodology}

- To model attached-nitroxide spin label membrane protein systems: CHARMM-GUI (1) web-based service was used and setting up systems by VMD software (2) (version 1.9.3). Spin label-attached KvAP-VSDs were embedded in a POPC lipid bilayer and solvated with TIP3P water molecules. The simulation box is approximately $84 \times 81 \times 90 \AA^{3}$ in size. $\mathrm{Na}^{+}$and $\mathrm{Cl}^{-}$were used as counterions.

- To perform MD simulations, NAMD program (3) (version 2.11) with CHARMM36 force fields (4) were employed. Switch and cutoff distances were 10 and $12 \AA$. Simulation time was $4 \mathrm{~ns}$ for each system at $298 \mathrm{~K}$ and $1 \mathrm{~atm}$. Time step was $1 \mathrm{fs}$. Trajectories were saved every 1000 fs. 


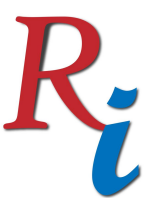

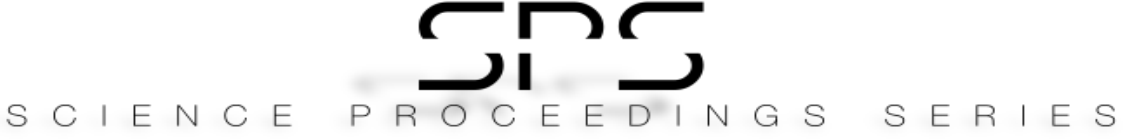

http://readersinsight.net/SPS

- To analyze the MD trajectories: Root-Mean-Square Fluctuation (RMSF), dihedral angles $\left(\chi_{1}-\chi_{5}\right)$ and $\mathrm{C} \alpha$ - nitroxide distance $(r)$ of spin label side chains were calculated. The obtained results were compared with experimental data from our collaborators.

\section{Results}

For each system, a spin label was covalently attaching to one amino acid of the X-ray structure of KvAP-VSD (5) by site-directed mutagenesis. Continuing this procedure for all amino acid positions (residues 20-151), there were a total of 132 obtained systems. It was found that the dynamics from RMSF calculations and mobilities data from EPR (6) had similar patterns as shown in graphical abstract. The loop sites were more flexible than the segment sites (S1-S4). Besides, the comparable tendencies can be seen for the dynamics of the backbones when attaching/without spin labels. As for the dihedral angles, they were ranged from $-180^{\circ}$ to +180 with a confidence interval of $\pm 30^{\circ}$. The $\chi_{1}$ rotations were equal to $\pm 60^{\circ}$ and $\pm 180^{\circ} . \chi_{2}$ angle values were located at $\pm 75^{\circ}$ and $\pm 180^{\circ}$. The $\chi_{3}$ configurations distributed at $90^{\circ}$ and $-90^{\circ} . \chi_{4}$ ranged from $\pm 60^{\circ}$ to $\pm 180^{\circ}$. For $\chi_{5}$, they spread from $0-120^{\circ}$. These distributions showed a good agreement with Tombolato F. and co-worker study (7). The variation of the nitroxide-C $\alpha$ distances $(r)$ were from 6.5 to $10 \AA$ whereas DeSensi, S. C., et. al. found it $\sim 7 \AA$ when attaching to T4 lysozyme (8).

\section{Findings}

We constructed structures of the cysteine-mutated membrane protein with the nitroxide spin label attaching at all amino acid sites. The SDSL technique and MD simulations are such a valuable combination to study the structure and dynamical properties of spin label side chain which are thoroughly sensitive to reflect the membrane protein conformational fluctuation.

\section{Acknowledgement}

Authors would like to thank Chulalongkorn University and ASEAN Scholarship for their continuing support.

\section{REFERENCES}

[1] Jo S, Kim T, Iyer VG, Im W. CHARMM-GUI: a web-based graphical user interface for CHARMM. J Comput Chem. 2008;29(11):1859-65.

[2] Humphrey W, Dalke A, Schulten K. VMD: visual molecular dynamics. J Mol Graph. 1996;14(1):33-8, 27-8. 


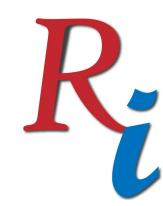

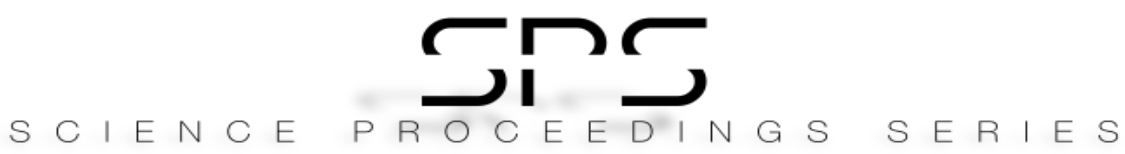

http://readersinsight.net/SPS

[3] Phillips JC, Braun R, Wang W, Gumbart J, Tajkhorshid E, Villa E, et al. Scalable molecular dynamics with NAMD. J Comput Chem. 2005;26(16):1781-802.

[4] Klauda JB, Venable RM, Freites JA, O'Connor JW, Tobias DJ, Mondragon-Ramirez C, et al. Update of the CHARMM all-atom additive force field for lipids: validation on six lipid types. J Phys Chem B. 2010;114(23):7830-43.

[5] Jiang Y, Lee A, Chen J, Ruta V, Cadene M, Chait BT, et al. X-ray structure of a voltagedependent K+ channel. Nature. 2003;423(6935):33-41.

[6] Li Q, Wanderling S, Sompornpisut P, Perozo E. Structural basis of lipid-driven conformational transitions in the KvAP voltage-sensing domain. Nat Struct Mol Biol. 2014;21(2):160-6.

[7] Tombolato F, Ferrarini A, Freed JH. Dynamics of the nitroxide side chain in spin-labeled proteins. J Phys Chem B. 2006;110(51):26248-59.

[8] DeSensi SC, Rangel DP, Beth AH, Lybrand TP, Hustedt EJ. Simulation of nitroxide electron paramagnetic resonance spectra from brownian trajectories and molecular dynamics simulations. Biophys J. 2008;94(10):3798-809.

Author's Biography

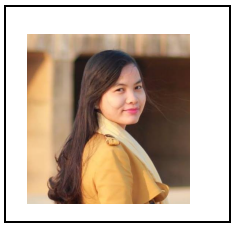

B.Ed.(Chemistry) Quy Nhon University

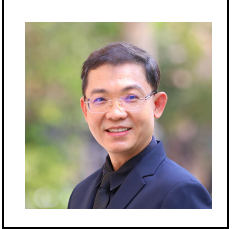

Professor Chulalongkorn University

Research Associate University of Chicago

Postdoctoral fellow University of Virginia

Ph.D. University of Florence, Italy

M.Sc. (Chemistry) Chulalongkorn University

B.Sc. (Chemistry) Chulalongkorn University. 